\title{
TRANSLATION INVARIANCE OF WEAK KAM SOLUTIONS OF THE NEWTONIAN $N$-BODY PROBLEM
}

\author{
EZEQUIEL MADERNA
}

(Communicated by James E. Colliander)

\begin{abstract}
We consider the Hamilton-Jacobi equation $H\left(x, d_{x} u\right)=c$, where $c \geq 0$, of the classical $N$-body problem in some Euclidean space $E$ of dimension at least two. The fixed points of the Lax-Oleinik semigroup are global viscosity solutions for the critical value of the constant $(c=0)$, also called weak KAM solutions. We show that all these solutions are invariant under the action by translations of $E$ in the space of configurations. We also show the existence of non-invariant solutions for the supercritical equations $(c>0)$.
\end{abstract}

\section{INTRODUCTION AND RESULTS}

1.1. Preliminaries. Let $E$ be a Euclidean vector space of dimension $k \geq 2$ in which $N$ punctual masses $m_{1}, \ldots, m_{N}>0$ are mutually attracted by the Newtonian gravitational forces. At each time, the position vectors of the $N$ bodies determine a configuration of the system $x=\left(r_{1}, \ldots, r_{N}\right) \in E^{N}$. The Newtonian potential is the function $U: E^{N} \rightarrow(0,+\infty]$ defined by

$$
U(x)=\sum_{1 \leq i<j \leq N} \frac{m_{i} m_{j}}{\left\|r_{i}-r_{j}\right\|} .
$$

It is clear that $U(x)<+\infty$ if and only if $x$ is a configuration without collisions, meaning that there are no values of $i<j$ such that $r_{i}=r_{j}$. Moreover, if we denote by $\Omega \subset E^{N}$ the open and dense subset of configurations without collisions, then the Newtonian potential is an analytical function on $\Omega$, and the law of gravitation is equivalent to the second order differential equation $\ddot{x}=\nabla U$ on $\Omega$, where the gradient is taken with respect to the mass inner product.

As well as the equations of motion, the Hamiltonian and the Lagrangian of the system admit a synthetic expression in terms of the mass inner product. We recall that given two configurations $x=\left(r_{1}, \ldots, r_{N}\right)$ and $y=\left(s_{1}, \ldots, s_{N}\right)$, their mass inner product is given by

$$
x \cdot y=\sum_{i=1}^{N} m_{i}\left\langle r_{i}, s_{i}\right\rangle .
$$

With the natural identification $T E^{N} \simeq E^{N} \times E^{N}$, the Lagrangian function of the system reads

$$
L(x, v)=\frac{1}{2}|v|^{2}+U(x)
$$

Received by the editors May 30, 2011 and, in revised form, November 7, 2011. 2010 Mathematics Subject Classification. Primary 37J15, 70H20, 49L25. 
where $|v|=(v \cdot v)^{1 / 2}$ denotes the induced norm by the mass inner product on $E^{N}$. Consequently, if $p=\left(p_{1}, \ldots, p_{N}\right)$ is in the dual space $\left(E^{N}\right)^{*} \simeq\left(E^{*}\right)^{N}$ and if $|p|$ denotes the operator norm of $p$ for the mass inner product, we have

$$
|p|^{2}=\sum_{i=1}^{N} \frac{1}{m_{i}}\left\|p_{i}\right\|^{2},
$$

where $\left\|p_{i}\right\|$ is the operator norm of $p_{i} \in E^{*}$. Since the Hamiltonian $H$ of the system is defined on each fiber of the cotangent bundle $T^{*} E^{N}$ as the convex dual of the Lagrangian, we obtain the expression

$$
H(x, p)=\frac{1}{2}|p|^{2}-U(x) .
$$

Recall that here the Legendre transform $\mathcal{L}(x, v)=\partial_{v} L(x, v) \in T^{*} E^{N}$ does not depend on the configuration $x$, hence it can be defined as a map $\mathcal{L}: E^{N} \rightarrow\left(E^{N}\right)^{*}$. Thus $p=\mathcal{L}(v)$ will mean that $p(w)=w \cdot v$ for all $w \in E^{N}$. It is easy to check the Young-Fenchel inequality, which assures that for all $p \in\left(E^{N}\right)^{*}$ and $v \in E^{N}$ we have $p(v) \leq H(x, p)+L(x, v)$ and that equality holds if and only if $p=\mathcal{L}(v)$.

Finally, we recall that if $M=m_{1}+\cdots+m_{N}$ is the total mass of the system, the center of mass of a configuration is given by the linear map $G: E^{N} \rightarrow E$ :

$$
G(x)=\frac{1}{M} \sum_{i=1}^{N} m_{i} r_{i} .
$$

For any vector $r \in E$ we have $x \cdot(r, \ldots, r)=\langle M G(x), r\rangle$; thus it is clear that the space of configurations decompose as the orthogonal direct sum $E^{N}=\mathcal{X} \oplus \Delta$, where $\mathcal{X}=\operatorname{ker} G$ is the subspace of all centered configurations and $\Delta$ is the diagonal subspace. We also recall that, since the sum of all forces acting on the system is zero, the center of mass of any motion has constant velocity.

1.2. The Hamilton-Jacobi equation and weak KAM solutions. Since our mechanical system is autonomous, its Hamiltonian function $H$ is time independent. Therefore the natural question which arises is the existence and characterization of the stationary solutions of the Hamilton-Jacobi equation $\partial_{t} S+H\left(x, \partial_{x} S\right)=0$, that is to say, solutions of the form $S(t, x)=u(x)-c t$. In this paper we will study the global stationary solutions, or in other words, functions $u: E^{N} \rightarrow \mathbb{R}$ satisfying the Hamilton-Jacobi equation $H\left(x, d_{x} u\right)=c$, more explicitly, the equation

$$
\frac{1}{2} \sum_{i=1}^{N} \frac{1}{m_{i}}\left\|\frac{\partial u}{\partial r_{i}}\right\|^{2}-\sum_{i<j} \frac{m_{i} m_{j}}{\left\|r_{i}-r_{j}\right\|}=c .
$$

Considering that the mutual distances between the bodies can be arbitrarily large, it is clear that equation (11) only has global solutions for values of the constant $c \geq 0$.

A classical global solution, or global smooth solution, is a continuous function $u: E^{N} \rightarrow \mathbb{R}$ whose restriction to the open and dense set of configurations without collisions $\Omega$ is of class $C^{1}$ and such that (1) is verified at each point of this set. Until now, the only known smooth global solutions are solutions for the case $N=2$, that is to say, for the Kepler problem in the space $E$. A direct computation shows that functions $u\left(r_{1}, r_{2}\right)= \pm\left\|r_{1}-r_{2}\right\|^{1 / 2}$ are global smooth solutions. 
Recently, the author has shown in [6] the existence of weak KAM solutions for a family of $N$-body problems with homogeneous potentials, including the Newtonian case. They are global viscosity solutions of the critical equation $H\left(x, d_{x} u\right)=0$. The existence was obtained by the application of a fixed point theorem to the action of the Lax-Oleinik semigroup on the convex set of viscosity subsolutions.

Moreover, if $\Gamma$ is a given symmetry group of the system, then the linear space of all invariant functions has non-trivial intersection with the set of subsolutions, since clearly constant functions are invariant subsolutions. Considering that the semigroup preserves the set of all invariant subsolutions, which is also convex, the existence of invariant weak KAM solutions is also obtained (Theorem 3 in [6]). It is evident that the application of a given isometry of the Euclidean space $E$ to each component of a configuration defines a symmetry of the system. Thus the translation group of $E$, as well as the orthogonal group $O(E)$, acts naturally on the space of configurations by symmetries. Depending on the values of the masses, other symmetry groups can act on the space of configurations. For example, in the non-generic case in which the masses of some bodies are equal, every permutation of the position vectors of these bodies is a symmetry.

We shall remark that for a smooth, convex and superlinear Hamiltonian function $H: T^{*} M \rightarrow \mathbb{R}$ on a compact manifold $M$, weak KAM solutions are always invariant under the action of the identity component of a compact symmetry group (see [5]).

Even if the orthogonal group is compact, there are always non-invariant solutions for the action of his identity component $S O(E)$. The critical Busemann functions explicitly computed in [6] for the Kepler problem in the plane with equal masses are examples of such non-invariant weak KAM solutions. Nevertheless, weak KAM solutions are always invariant under translations.

Theorem 1. Every weak KAM solution of the Newtonian $N$-body problem in a Euclidean space $E$ of dimension at least two is translation invariant. More precisely, given a weak $K A M$ solution $u: E^{N} \rightarrow \mathbb{R}$, we have that

$$
u\left(r_{1}, \ldots, r_{N}\right)=u\left(r_{1}+r, \ldots, r_{N}+r\right)
$$

for every configuration $x=\left(r_{1}, \ldots, r_{N}\right) \in E^{N}$ and every $r \in E$.

The hypothesis $\operatorname{dim} E \geq 2$ will only be used in order to apply Marchal's theorem (whose proof in the case $\operatorname{dim} E>3$ is due to D. Ferrario and S. Terracini) to the calibrated curves of a given solution. This said, there is no evidence that suggests the theorem is not valid in the one dimensional case.

We will show that the translation invariance of a weak KAM solution is equivalent to the fact that all its calibrated curves are motions with fixed center of mass (Proposition 4). On the other hand, a very strong property of the calibrated curves of a weak KAM solution is the free time minimization property, which we recall below. Therefore, the proof of Theorem 1 will follow from Lemma 3 , where Marchal's theorem is applied. The dynamics when $t \rightarrow+\infty$ of the free time minimizers of the Newtonian $N$-body problem is described in [1, where it is proved that motions of this kind are completely parabolic, meaning that the velocities of the bodies must tend to zero.

The following corollary will be easily deduced from the theorem. It shows as a counterpart that the supercritical equations (1), that is, for the values of $c>0$, can be non-invariant under translations. 
Corollary 2. Given a weak KAM solution $u_{0}: E^{N} \rightarrow \mathbb{R}$ of the $N$-body problem and an arbitrary vector $r \in E$, the function

$$
u_{r}(x)=u_{0}(x)+\langle M G(x), r\rangle
$$

is a global viscosity solution of the Hamilton-Jacobi equation

$$
H\left(x, d_{x} u\right)=\frac{1}{2} M^{*}\|r\|^{2},
$$

where $M^{*}=\sum_{i=1}^{N} m_{i}^{-1}$.

The following section is devoted to prove the above results. It is interesting to remark that all the statements and proofs remains valid if we replace the Newtonian potential by homogeneous potential of degree $\alpha \in(-2,0)$.

\section{ProOfs}

We start by recalling some basic notions of the calculus of variations that will be used. The Lagrangian action of an absolutely continuous curve $\gamma:[a, b] \rightarrow E^{N}$,

$$
A(\gamma)=\int_{a}^{b} L(\gamma(t), \dot{\gamma}(t)) d t
$$

is a well defined function of $\gamma$ taking values in $(0,+\infty]$. For two given configurations $x, y \in E^{N}$, the set of all absolutely continuous curves starting at $x$ and arriving at $y$ in time $T>0$ will be denoted $\mathcal{C}(x, y, T)$. It is well known that the Lagrangian action is lower semicontinuous, which implies that the infimum of its restriction to any set $\mathcal{C}(x, y, T)$ is always reached. In what follows, $\phi(x, y, T)$ will denote the minimum of the Lagrangian action restricted to $\mathcal{C}(x, y, T)$.

Let us recall Marchal's theorem on the fixed endpoint problem (cf. [2, 44). It says that minimizers of the Lagrangian action in a set $\mathcal{C}(x, y, T)$ do not suffer collisions in any interior time. In other words, if an absolutely continuous curve $\gamma:[a, b] \rightarrow E^{N}$ is such that $A(\gamma) \leq A(\sigma)$ for every $\sigma \in \mathcal{C}(\gamma(a), \gamma(b), b-a)$, then the curve $\gamma$ avoids collisions except perhaps in the prescribed endpoints; that is to say, $\gamma(t) \in \Omega$ for all $t \in(a, b)$. In particular, the restriction of a such curve to the open interval $(a, b)$ satisfies the equation of motion, hence it must be of class $C^{\infty}$. Furthermore, if one of its endpoints, say $\gamma(a)$, is a configuration without collisions, then the solution can be extended to an interval of the form $(a-\epsilon, b)$ for some $\epsilon>0$, which implies that $\dot{\gamma}(a)$ exists and coincides with $\lim _{t \rightarrow a^{+}} \dot{\gamma}(t)$.

2.1. Action potential, free time minimizers and calibrated curves. We will recall some results from [6] as well as some well known features of weak KAM theory.

2.1.1. The action potential. The set of all absolutely continuous curves starting at $x$ and ending at $y$ without restriction of time will be denoted $\mathcal{C}(x, y)$. The action potential, also called the Mañe critical potential in Aubry-Mather theory, is the function $\phi: E^{N} \times E^{N} \rightarrow \mathbb{R}^{+}$:

$$
\phi(x, y)=\inf \{A(\gamma) \mid \gamma \in \mathcal{C}(x, y)\}=\inf _{T>0} \phi(x, y, T) .
$$

For convenience, we will use the maximum norm in $E^{N}$,

$$
\left\|\left(r_{1}, \ldots, r_{N}\right)\right\|=\max _{i=1, \ldots, N}\left\|r_{i}\right\| .
$$


The action potential is a distance function in the space of configurations. Moreover, it is Hölder continuous in each variable with respect to any norm in $E^{N}$. In fact, there is a constant $\eta>0$ such that

$$
\phi(x, y) \leq \eta\|x-y\|^{1 / 2}
$$

for every pair of configurations. The constant $\eta$ depends only on the number of bodies $N$ and the total mass $M$. Since several of the notions considered here are easily characterized in terms of the action potential, it will play a central role.

Another important property of the action potential that we will also use is that it is locally Lipschitz in $\Omega \times \Omega$. More precisely, if $C \subset \Omega$ is a compact set, then there is a constant $K>0$ such that $\phi(x, y) \leq K\|x-y\|$ whenever $(x, y) \in C \times C$. Of course the Lipschitz constant grows, as well as the compact set $C$ approaches, some given collision configuration.

2.1.2. Free time minimizers. We call a free time minimizer, or semistatic curve, an absolutely continuous curve such that the action of each compact segment equals the action potential between the endpoints of the segment. In other words, a curve $\gamma: I \rightarrow E^{N}$ is a free time minimizer if we have $A\left(\left.\gamma\right|_{[a, b]}\right)=\phi(\gamma(a), \gamma(b))$ whenever $[a, b] \subset I$ is a compact subinterval. When $\operatorname{dim} E \geq 2$, Marchal's theorem implies that a free time minimizer defined on an interval $[a, b]$ avoids collisions for $t \in(a, b)$.

2.1.3. Viscosity subsolutions as dominated functions. On the other hand, we will be concerned with the notion of weak solution of (1) introduced by M. Crandall and P.-L. Lions in 3], namely the notion of viscosity solution. The classical way to define them is in terms of test functions, as functions which are at the same time viscosity subsolutions and viscosity supersolutions. It is well known that the set of viscosity subsolutions of the critical Hamilton-Jacobi equation $H\left(x, d_{x} u\right)=0$, which we call the set of dominated functions, is exactly the convex set

$$
\mathcal{H}=\left\{u: E^{N} \rightarrow \mathbb{R} \mid u(y)-u(x) \leq \phi(x, y) \text { for all } x, y \in E^{N}\right\} .
$$

By the previous considerations, we know in particular that functions in $\mathcal{H}$ are Hölder continuous and locally Lipschitz in $\Omega$. Since $\Omega$ is a set of total measure, by Rademacher's theorem we also know that they are differentiable almost everywhere.

2.1.4. Calibrated curves. For a given dominated function $u \in \mathcal{H}$, we say that an absolutely continuous curve $\gamma: I \rightarrow E^{N}$ is calibrated for $u$ if for each compact subinterval $[a, b] \subset I$ we have that $u(\gamma(b))-u(\gamma(a))=A\left(\left.\gamma\right|_{[a, b]}\right)$. Note that a calibrated curve is always a free time minimizer. On the other hand, if $u \in \mathcal{H}$ and $\gamma$ is a calibrated curve for $u$, defined on $I=(a, b)$ and of class $C^{1}$, then $u$ is differentiable at $\gamma(t)$ for all $t \in(a, b)$, and $d_{\gamma(t)} u=\mathcal{L}(\dot{\gamma}(t))$. It follows, using the Young-Fenchel inequality, that $H\left(\gamma(t), d_{\gamma(t)} u\right)=0$ for all $t \in(a, b)$.

2.1.5. Characterization of weak KAM solutions. The action of the Lax-Oleinik semigroup $\left(T_{t}\right)_{t \geq 0}$ is well defined in $[0,+\infty) \times \mathcal{H}$ by

$$
T_{t} u(x)=\inf \left\{u(y)+\phi(y, x, t) \mid y \in E^{N}\right\}
$$

for $t>0$ and $T_{0}=i d_{\mathcal{H}}$. A weak KAM solution is a fixed point of the Lax-Oleinik semigroup, that is to say, a function $u \in \mathcal{H}$ such that $T_{t} u=u$ for all $t \geq 0$. It is proved in [6] that such fixed points exist and that, in fact, they are global viscosity solutions of the critical Hamilton-Jacobi equation. Moreover they are characterized between dominated functions as follows: $u \in \mathcal{H}$ is a weak KAM solution if and only 
if for each configuration $x \in E^{N}$ there is a calibrated curve $\gamma_{x}:[0,+\infty) \rightarrow E^{N}$ such that $\gamma_{x}(0)=x$. By all the above considerations, we deduce that if $u$ is a weak KAM solution differentiable at a configuration $x \in \Omega$, then there is only one calibrated curve, since it must coincide with the motion generated by the initial conditions $\gamma(0)=x$ and $\dot{\gamma}(0)=\mathcal{L}^{-1}\left(d_{x} u\right)$.

2.2. Proof of Theorem 1. As we have said, the proof is a direct consequence of the following lemma and proposition.

Lemma 3. If $\operatorname{dim} E \geq 2$ and $x:[0,+\infty) \rightarrow E^{N}$ is a free time minimizer, then the center of mass $G(x(t))$ is constant.

Proof. First, we must observe that Marchal's theorem implies $x(t) \in \Omega$ for all $t>0$. Therefore $x$ satisfies the equation of motion on $(0,+\infty)$, from which we deduce that $G(x(t))$ has constant velocity, even at $t=0$. With the notation $x_{0}=x(0)$ and $G_{0}=G\left(x_{0}\right)$, we have that $G(x(t))$ is of the form $G_{0}+t v$ for some vector $v \in E$. We will prove that $v=0$.

Let $y:[0,+\infty) \rightarrow E^{N}$ be the internal motion associated with $x$, that is to say, the curve defined by $x(t)=y(t)+\delta\left(G_{0}+t v\right)$, where $\delta: E \rightarrow E^{N}$ is the map $\delta(r)=(r, \ldots, r)$. It is clear that for all $t \geq 0$ we have that $U(x(t))=U(y(t))$ and $G(y(t))=0$. On the other hand, a computation shows that $\dot{x} \cdot \dot{x}=\dot{y} \cdot \dot{y}+M\|v\|^{2}$ for all $t>0$. Hence we deduce that for all $T>0$ we have

$$
A\left(\left.x\right|_{[0, T]}\right)=A\left(\left.y\right|_{[0, T]}\right)+\frac{1}{2} T M\|v\|^{2} .
$$

Since $x$ is a free time minimizer and $\phi$ verifies the triangle inequality, we also obtain

$$
A\left(\left.x\right|_{[0, T]}\right)=\phi\left(x_{0}, x(T)\right) \leq \phi\left(x_{0}, x_{0}+T \delta(v)\right)+\phi\left(x_{0}+T \delta(v), x(T)\right) .
$$

Let $y_{T}$ be the curve obtained by translating the restriction of $y$ to the interval $[0, T]$ in such a way that the center of mass of $y_{T}$ becomes fixed at $G(x(T))$. Thus, $y_{T}$ is the curve defined for $t \in[0, T]$ by $y_{T}(t)=y(t)+\delta\left(G_{0}+T v\right)$. Both curves have the same action, but now we have $\gamma_{T} \in \mathcal{C}\left(x_{0}+T \delta(v), x(T)\right)$. Therefore,

$$
\phi(x(0)+T \delta(v), x(T)) \leq A\left(\left.y\right|_{[0, T]}\right) .
$$

We now use the Hölder estimation for the action potential, and we get the bound

$$
\phi(x(0), x(0)+T \delta(v)) \leq \eta\|T \delta(v)\|^{1 / 2}=T^{1 / 2} \eta\|v\|^{1 / 2} .
$$

We can conclude that the inequality

$$
\frac{1}{2} T M\|v\|^{2} \leq T^{1 / 2} \eta\|v\|^{1 / 2}
$$

holds for all $T>0$, but this is possible only in the case $v=0$.

Proposition 4. A weak KAM solution $u: E^{N} \rightarrow \mathbb{R}$ is invariant under translations if and only if all calibrated curves have constant center of mass.

Proof. We start by showing the necessity of the condition. Let $u$ be a weak KAM solution which is invariant under translations. At each point of differentiability $x \in E^{N}$ we must have $\Delta \subset \operatorname{ker} d_{x} u$. Let $x \in E^{N}$ be any configuration, and let $\gamma_{x}:[0,+\infty)$ be a calibrated curve for $u$ starting at $x$. We know that $u$ is differentiable at $\gamma_{x}(t)$ for all $t>0$ and that $d_{\gamma_{x}(t)} u=\mathcal{L}\left(\dot{\gamma}_{x}(t)\right)$; that is to say, $d_{x} u(w)=w \cdot \dot{\gamma}_{x}(t)$ for all $w \in E^{N}$. It follows that $\dot{\gamma}_{x}(t) \in \Delta^{\perp}=\mathcal{X}$ for $t>0$, hence that $G\left(\gamma_{x}(t)\right)$ is constant. 
Now let $u$ be a weak KAM solution such that all calibrated curves have constant center of mass. Let $x \in E^{N}$ be some given configuration, and choose a non-zero vector $r \in E$. We will prove that $u(x)=u(x+\delta(r))$, where $\delta(r)=(r, \ldots, r) \in \Delta$.

Since $u$ is continuous and the set $\Omega$ is dense and translation invariant, it suffices to consider configurations $x \in \Omega$. Let

$$
B(\epsilon, r)=\left\{z \in E^{N} \mid z \cdot \delta(r)=0,\|z\| \leq \epsilon\right\}
$$

be the closed ball of radius $\epsilon>0$ in the subspace of $E^{N}$ orthogonal to $\delta(r) \in \Delta$. Since $x \in \Omega$, we can assume that $\epsilon$ is so small that the set

$$
B=\{x+z \mid z \in B(\epsilon, r)\}
$$

is contained in $\Omega$, which also implies that the compact cylinder

$$
C=\{y+t \delta(r) \mid y \in B, t \in[0,1]\}
$$

is contained in $\Omega$. Let us call $C^{\prime} \subset C$ the set of points where $u$ is differentiable. Recall that $u$ is a dominated function; hence it is a Lipschitz function on $C$, and $C^{\prime}$ has total measure in $C$. For $q \in C^{\prime}$, let $\gamma_{q}:[0,+\infty) \rightarrow E^{N}$ be the only calibrated curve for $u$ with $\gamma_{q}(0)=q$. We know that $d_{q} u(w)=w \cdot \dot{\gamma}_{q}(0)$ for all $w \in E^{N}$. By using the hypothesis we deduce that $\dot{\gamma}_{q}(0) \in \mathcal{X}=\operatorname{ker} G=\Delta^{\perp}$ and therefore that $\Delta \subset \operatorname{ker} d_{q} u$ for all $q \in C^{\prime}$.

We now consider the Lipschitz continuous function $f: B \times[0,1] \rightarrow \mathbb{R}$ defined by $f(y, t)=u(y+t \delta(r))-u(y)$. It is clear that $\partial_{t} f$ is zero almost everywhere. Therefore, applying Fubini's theorem $\partial_{t} f$ on a cylinder of the form $A \times[0,1]$, where $A \subset B$ is an open set, we obtain

$$
\int_{A} f(y, 1) d y=0
$$

Since the open subset $A \subset B$ is arbitrary, we must have $f(y, 1)=0$ for all $y \in B$. Hence, we have $f(x, 1)=0$; that is to say, $u(x+\delta(r))=u(x)$.

\subsection{Viscosity solutions of the supercritical equations.}

Proof of Corollary 2. Let $u_{0}$ be a weak KAM solution and $r \in E$. We will use the fact that $u_{0}$ is a global solution of $H\left(x, d_{x} u\right)=0$ in the viscosity sense. As before, we call $\delta: E \rightarrow \Delta$ the trivial isomorphism. Thus the function $u_{r}$ can be written as $u_{r}=u_{0}+a_{r}$, where $a_{r}$ is the linear function $a_{r}(x)=x \cdot \delta(r)$.

Let $x_{0}$ be a given configuration, and suppose that $\varphi \in C^{1}\left(E^{N}\right)$ is such that $\varphi-u_{r}$ has a local minimum at $x_{0}$. Since $u_{0}$ is a viscosity solution of $H\left(x, d_{x} u\right)=0$ and we have that $\left(\varphi-a_{r}\right)-u_{0}$ has a local minimum at $x_{0}$, we have $H\left(x_{0}, d_{x_{0}}\left(\varphi-a_{r}\right)\right) \leq 0$.

On the other hand, by Theorem 1 we know that $u_{0}$ is translation invariant; hence $\Delta \subset \operatorname{ker} d_{x_{0}}\left(\varphi-a_{r}\right)$. Since $d_{x_{0}} a_{r}=a_{r}$, it is clear that $\mathcal{X} \subset \operatorname{ker} d_{x_{0}} a_{r}$ and that $\left|d_{x_{0}} a_{r}\right|^{2}=M^{*}\|r\|^{2}$, where $M^{*}=m_{1}^{-1}+\cdots+m_{N}^{-1}$. Recalling that $\Delta^{\perp}=\mathcal{X}$, we deduce that

$$
\left|d_{x_{0}} \varphi\right|^{2}=\left|d_{x_{0}}\left(\varphi-a_{r}\right)\right|^{2}+M^{*}\|r\|^{2}
$$

and we conclude that

$$
H\left(x_{0}, d_{x_{0}} \varphi\right)=H\left(x_{0}, d_{x_{0}}\left(\varphi-a_{r}\right)\right)+\frac{1}{2} M^{*}\|r\|^{2} \leq \frac{1}{2} M^{*}\|r\|^{2},
$$

proving that $u_{r}$ is a viscosity subsolution of the supercritical equation at $x_{0}$. With a symmetric argument it can be proved that $u_{r}$ is a viscosity supersolution of the same equation. 


\section{REFERENCES}

1. A. Da Luz and E. Maderna, On the free time minimizers of the Newtonian $N$-body problem, preprint, http://premat.fing.edu.uy/2009.htm

2. A. Chenciner, Action minimizing solutions of the Newtonian n-body problem: From homology to symmetry, Proceedings of the ICM, Vol. III (Beijing, 2002), 279-294, Higher Ed. Press, Beijing, 2002. MR1957539(2004f:70026a)

3. M.G. Crandall and P.-L. Lions, Viscosity solutions of Hamilton-Jacobi equations, Trans. Amer. Math. Soc. 277 (1983), 1-42. MR690039 (85g:35029)

4. D. Ferrario and S. Terracini, On the existence of collisionless equivariant minimizers for the classical n-body problem, Invent. Math. 155 (2004), no. 2, 305-362. MR2031430 (2005b:70010)

5. E. Maderna, Invariance of global solutions of the Hamilton-Jacobi equation, Bull. Soc. Math. France 130 (2002) no. 4, 493-506. MR1947450(2004b:37132)

6. E. Maderna, On weak KAM theory for $N$-body problems, Ergodic Theory Dynam. Systems 32 (2012), no. 3, 1019-1041. MR2995654

CMAt, Universidad de la República, Iguá 4225, 11400 Montevideo, Uruguay

E-mail address: emaderna@cmat.edu.uy 